\title{
A Survey on Context-Aware Middleware
}

\author{
Chitra G. Sahu ${ }^{1}$, Dr. D. S. Adane ${ }^{2}$ \\ Research Scholar, Department of Computer Science and Engineering, ShriRamdeobaba College of Engineering and \\ Management, Nagpur, India. ${ }^{1}$ \\ Head of Department, Department of Information Technology, ShriRamdeobaba College of Engineering and \\ Management, Nagpur, India. ${ }^{2}$
}

\begin{abstract}
The middleware deals with different functionality such as service discovery, asynchronous messaging, publish/subscribe event management,managingand storage of context information, constructing the user interface and management of the local and network resources. Also, it allows controlling the connectivity of the device; the middleware is proficient to switch traffic from one network connection to another.

In this literature review paper, we are presenting the experience of several research papers related to the next generation of middleware systems. Our work is concerned with providing basic understanding about the current requirements of mobile computing systems and how they can be achieved by modifying the existing middleware systems. Initially, we have defined Mobile Computing, its functions and different Mobile Platforms. Further, we have defined middleware and described its common features. Purposely, we have indicated the major challenges in mobile computing systems and analyzed the requirements of mobile computing. Following this, we review the different categories of mobile middleware technologies and show their relative strengths and weaknesses. On a broader perspective, we have tried to identify the major requirements for context-aware middleware systems and discussed how context-aware middleware can resolve the major issues in the development of application for pervasive computing. Also, we have highlighted the latest trend in context-aware computing; Internet of Things (IoT).
\end{abstract}

Keywords: Mobile Computing; Middleware; Context-Aware Middleware; Internet of Things.

\section{INTRODUCTION}

The availability of lightweight, portable computers and There is a great demand for designing modern middleware wireless technologies has created a new class of systems that can support new requirements imposed by applications called mobile applications, presenting mobility and as context aware middleware deals with the challenging problems to the designers. These applications current context of the user, we found in our survey that it often run on scarce resource platforms such as personal is the only middleware that can meet the changing digital assistants, notebooks, and mobile phones, each of requirements as it can adapt itself according to the which have limited CPU power, memory, and battery life. changing context. Thus we have focused our survey on They are usually connected to wireless links, which are context-aware middleware as it is the most efficient characterized by lower bandwidths, higher error rates and middleware for mobile computing systems.

more frequent disconnections. To support designers In this paper Section II defines middleware and gives building mobile applications, research in the field of common features and functions of middleware. In Section middleware systems has proliferated. Middleware aims at III we present a detailed description of context-aware facilitating communication and coordination of distributed middleware, defines context describes its life cycle and components, concealing complexity raised by mobility highlights main characteristics of context information; from application developers as much as possible. Most Section IV focuses on the issues related to context-aware distributed applications and services were designed with middleware. Section V points out future directions of the assumption that the terminals were powerful and research in the area of context-aware middleware for connected to fixed networks. Conventional middleware mobile computing and Section VI concludes the paper.

technologies distribution and thus, have focused on concealingthe problems of heterogeneity to enable the development of distributed systems. They let the

\section{MidDlewares}

application developers to focus on application complexity of distributed applications. These applic functionality rather than on handling explicitly with usually operate in an environment that may include distribution issues. Traditional middleware systems such heterogeneous computer architectures, operating systems, as CORBA, DCOM and Java RMI have shown their network protocols, and databases. It is unpleasant for an suitability for standard client-server applications.

application developer to deal with such heterogeneous However, under the highly variable computing plumbing. Middleware's primary role is to conceal this environmental conditions that characterize mobile complexity from developers by deploying an isolated layer platforms, it is believed that existing traditional of APIs. This layer bridges the gap between application middleware systems are not capable of providing adequate program and platform dependency.

support for the mobile wireless computing environment. Middleware is defined as follows by Linthicum D.[1] - 
"Middleware is an enabling layer of software that resides between the application program and the networked layer of heterogeneous platforms and protocols. It decouples applications from any dependencies on the plumbing layer that consists of heterogeneous operating systems, hardware platforms and communication protocols".

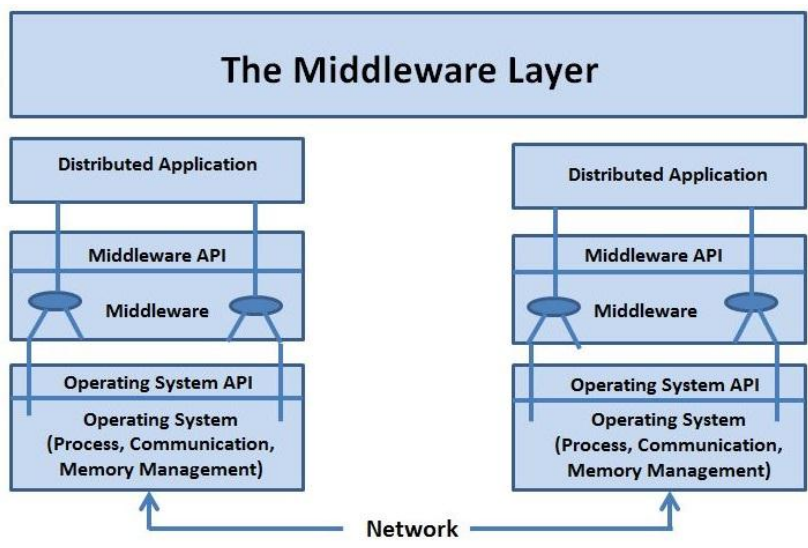

Fig 1.Middleware Layer

\section{A. Common Features of Middleware}

The following common features of wireless middleware products are needed to support mobile computing applications [2]:

Connection and message delivery: Middleware helps establish connections between mobile clients and servers over wireless networks and delivers messages over the connection. It also stores and forwards messages if the user is disconnected from the network.

Transformation: The middleware transforms data from one format to another (e.g., HTML to WML). The transformation may be intelligent enough to transform different types of data to different types of devices.

Detection and storage: Wireless middleware products can detect and store mobile device characteristics in a database. Upon detecting the type of mobile device or channel being used, the middleware can optimize the wireless data output according to device attributes.

Optimization: Middleware products can compress data to minimize the amount of data being sent over a slow cellular wireless link.

Security: Security features can be imbedded in wireless middleware to ensure end-to-end security. For example, digital certificates for handheld devices can be managed by a middleware service.

Operation support: Middleware can offer network and systems management utilities and tools to allow monitoring and troubleshooting wireless devices and networks.

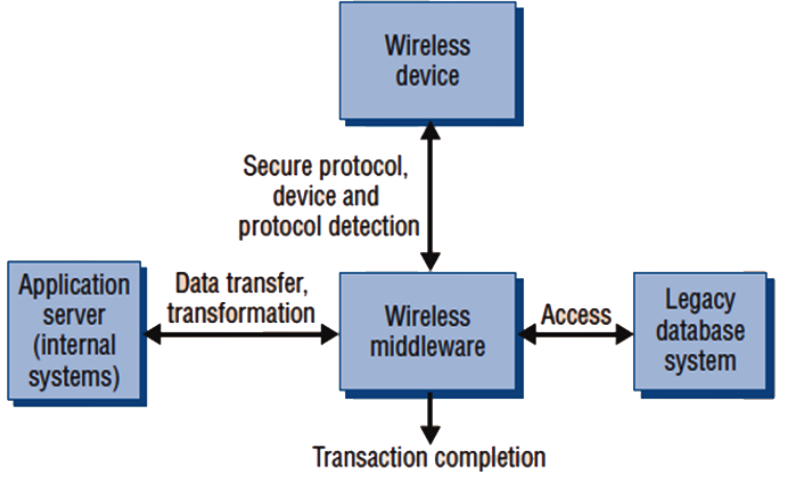

Fig 2. Functions of Wireless Middleware

The figure shows how middleware allows device, application server and database to work together as a mobile application. It also identifies the device and the communication protocol used which lets other parties know how to communicate with the device, it gives database and device a transparent route to each other's data and it also provide API to the application server for working with the device.

\section{III.CONTEXT-AWARE MIDDLEWARE}

Mobile systems run in an extremely dynamic environment. The execution context changes frequently due to the user's mobility. Mobile hosts often roam around different areas, and services that are available before disconnecting may not be available after reconnecting. Also, the bandwidth and connectivity quality may quickly alter based on the mobile host movements and their locations. The application developers cannot predict all the possible execution contexts that allow the application to know how to react in every scenario. The middleware has to expose the context information to the application to make it aware of the dynamic changes in execution environment. The application then instructs the middleware on how to adapt its own behavior in order to achieve the best quality of service. Many research groups gave special attention in particular to location awareness. For example, location information was exploited to provide travelers directional guidance, to discover neighboring services and to broadcast messages to users in a specific area. Most location-aware systems depend on the underlying network operating system to obtain location information and generate a suitable format to be used by the system. The heterogeneity of coordination information is not supported and hence different positioning systems are required to deal with different sensor technologies, such as the Global Positioning System (GPS) outdoors, and infrared and radio frequency indoors.

Context awareness involves performing data acquisition from sensors, context recognition and other tasks necessary to complete before the context can actually be used. Delegating the data acquisition and context processing tasks to applications makes them almost impossible to reuse. One solution to such a problem is to decouple the tasks from applications and move desired functionality to the lower layers. Such layers, which serve the needs of applications, usually form a special layer called middleware. This middleware layer hides the 
heterogeneity and distributed nature of devices measuring data fusion operations (e.g. GPS sensor readings as the context information. A context-aware middleware location information).

serves the context needs of applications.

Secondary context: Any information that can be computed A context-aware middleware has to provide the using primary context. The secondary context can be applications with the following context-oriented computed by using sensor data fusion operations or data functionality [3]:

- $\quad$ support of a variety of sensor devices retrieval operations such as web service calls (e.g. identify the distance between two sensors by applying sensor data

- support of the distributed nature of context fusion operations on two raw GPS sensor values). Further, information, because the data comes from retrieved context such as phone numbers, addresses, email different sources

- providing for transparent interpretation applications and abstraction of context data

- maintenance of context storage

- control of the context data flow

Mobility introduces a number of constraints to the middleware [4]:

- the bandwidth is low and hosts can be unreachable due to network partitions or poor coverage

- the local resources like memory capacity and CPU power of the device are very limited

- the communication between the system components is asynchronous

- the execution environment is dynamic

Current Context Aware Middleware free developers from the implementation of low-level details related to the network, like Concurrency Control, Transaction management, Network Communication, so focus is on application requirements. Modern distributed applications need a middleware that is capable of adapting to environment changes.

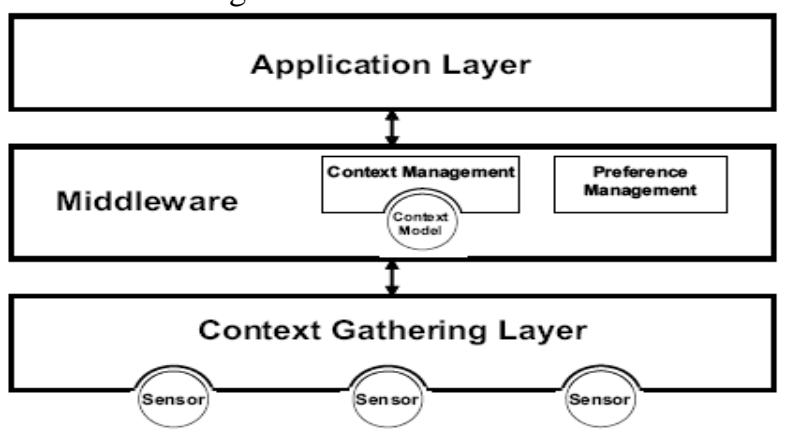

Fig 3. Architecture of a context-aware system

As per Marco B. and Leonardo B. [6], Context-awareness involves acquisition of contextual information, reasoning about context and modifying one's behavior based on the current context. A middleware for context awareness would provide support for each of these tasks. It would also define a common model of context, which all agents can use in dealing with context. It would also ensure that different agents in the environment have a common semantic understanding of contextual information. addresses, birthdays, list of friends from a contact of information provider based on a personal identity as the primary context can also be identified as secondary context.

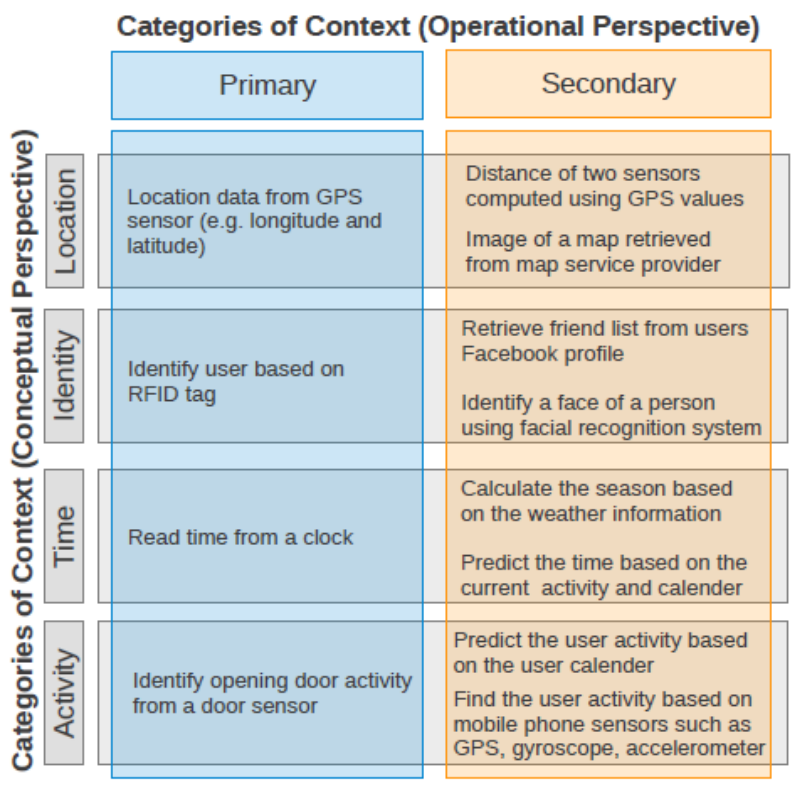

Fig 4. Context categorization in two different perspectives: conceptual and operational

With advances in technology sensors are being deployed at a significant rate. It generate enormous amount of raw data, all the raw data gathered from sensors are understood and added value by their collection, modelling, distribution and reasoning about the context. Huebscher M. C., McCann J. A., [9] has given the layers involved in context provision and is as depicted in the figure below:

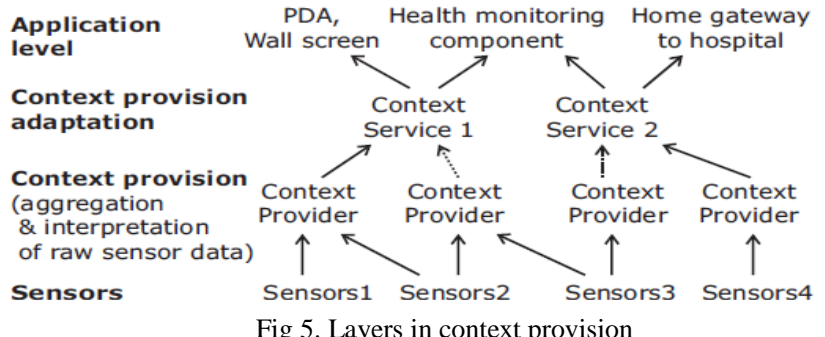

Sensors are at the bottom layer todeliver raw sensor data. These could be wireless sensor networks, ultrasonic "A context attribute is an element of the context model badges for location, RFID tags for identification, video describing the context. A context attribute has an cameras for tracking, or others. These produce raw sensor identifier, a type and a value, and optionally a collection of properties describing specific characteristics [7]."

As per Perera C. et al.[8], there are two main forms of context that are often used by the applications-

data, often preprocessed for saving communication cost as much as the sensor devices allow. These data are passed Context providers (CPs) are components (software or Primary context: Any information retrieved without using hardware) that aggregate and interpret the sensor data to existing context and without performing any kind of sensor produce some higher-level context, e.g. location, identity, 
type of activity, health condition of a person. As illustrated distributed to the consumers who are interested in context in the figure, more than one CP may access the same group (context dissemination).

of sensors. Similarly, it is possible for one CP to use sensor data from a group of sensors, as data redundancy often improves the total reliability of the derived context.

Context services (CSs) connects below to different context Sense: The data is sensed through sensors, including the providers that provide the same type of context, e.g. sensed data stored in databases (e.g. retrieve temperature location, but implemented using different underlying from a sensor, retrieve appointments details from a sensors, and above connect to the applications. They allow calendar).

an application to use a type of context while abstracting Derive: The information is generated by performing from the actual instance of a context provider. An computational operations on sensor data. These operations application can poll for context information or subscribe could beas simple as web service calls or as complex as for notification when a certain condition on the context mathematical functions run over sensed data (e.g. calculate information is met, e.g. when the temperature exceeds distance between two sensors using GPS coordinates). The $26^{\circ} \mathrm{C}$ or when Alice enters the kitchen.

necessary data should be available to apply any numerical Certain CPs and applications may use context information or logical reasoning technique.

from a CS to provide higher-level context. For instance, a Manually provided: Users provide context information CS may provide location in the form of $2 \mathrm{D}$ or 3D manually via predefined settings options such as coordinates in the home. A CP may then use this preferences. This method can be used to retrieve any type information to determine the room in the home this of information.

location maps to. Again, this higher-level context would be delivered through a context service.

\section{A. Context Life Cycle}

Dey provided a definition for context as follows [5]:

"Context is any information that can be used to by a given context-aware application is usually explicilly characterize the situation of an entity. An entity is a specified by the application developer, but may evolve person, place, or object that is considered relevant to the over time [7]." Context modelling is also widely referred interaction between a user and an application, including to as context representation. Typically, there are two steps the user and applications themselves."

This section shows the movement of context in context- Context modelling process: In the first step, new context aware systems. In addition to the life cycles, [10] information needs to be defined in terms of attributes, identified three phases in a typical context management characteristics, relationships with previously specified system: context acquisition, information processing, and context, quality-of context attributes and the queries for reasoning and decision. Perera C.et al. [8] derived an synchronous context requests.

appropriate (i.e. minimum number of phases but includes Organize context according to the model: In the second all essential) context life cycle as depicted in Figure 6. step, the result of the context modelling step needs to be This is the simplest form of a context life cycle. These four validated. Then the new context information needs to be steps are essential in context management systems and merged and added to the existing context information middleware solutions.

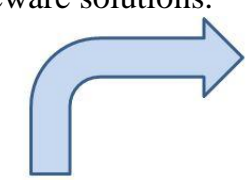

Context
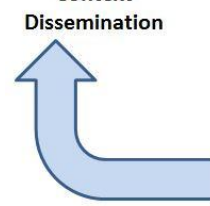
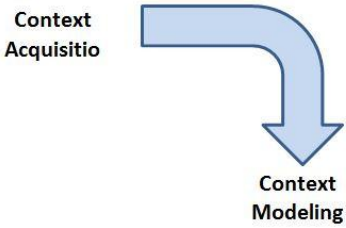

Context

Reasoning

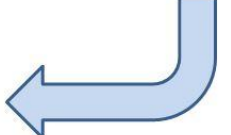

Fig 6. Context life cycle repository. Finally, the new context information is made available to be used when required.

3) Context Reasoning:

Context reasoning can be defined as a method of deducing new knowledge, and understanding better, based on the available context [11]. It can also be explained as a process of giving high-level context deductions from a set of contexts [12]. The requirement of reasoning also emerged due to two characteristics of raw context: imperfection (i.e. unknown, ambiguous, imprecise, or erroneous) and uncertainty. Context reasoning is broadly divided into three phases.[13]

This context life cycle consists of four phases. First, context needs to be acquired from various sources. The sources could be physical sensors or virtual sensors (context acquisition). Second, the collected data needs to be modeled and represent according to a meaningful manner (context modeling). Third, modeled data needs to be processed to derive high-level context information from low-level raw sensor data (context reasoning). Finally, both high-level and low-level context needs to be many years. network communication, collected data may be not accurate or missing. Therefore, data needs to be cleaned by filling missing values, removing outliers, validating context via multiple sources, and many more. These tasks have been extensively researched by database, data mining, and sensor network research communities over 
Sensor data fusion: It is a method of combining sensor take these problems into account to enable proper data from multiple sensors to produce more accurate, more reasoning about context information changes to achieve complete, and more dependable information that could not appropriate adaptations for the application, and thus be achieve through a single sensor [14]. In the IoT, fusion provide an experience for the user that is consistent with is extremely important, because there will be billions of the physical world.

sensors available. As a result, a large number of alternative Reasoning:Context-aware applications use context sources will exist to provide the same information. information to evaluate whether there is a change to the Context inference: Generation of high-level context user and/or to the environment situation; taking a decision information using lower-level context. The inferencing can whether any adaptation to that change is necessary often be done in a single interaction or in multiple interactions. requires reasoning capabilities. Reasoning techniques can For example, in the first iteration, longitude and latitude also be adopted to derive higher level context information. values of a GPS sensor may be inferred as PurplePickle cafe in canberra. In the next iteration PurplePickle café in canberra may be inferred as John's favourite cafe. Each iteration gives more accurate and meaningful information.

4) Context Distribution:

\section{IV.ISSUES WITH CONTEXT-AWARE MIDDLEWARE}

With the birth of context-aware middleware many issues are came out, like security, balance of user control, threat to privacy of the user. In the following sections we

Context distribution is a straight forward task. It examine the issues with the new middleware approach. provides methods to deliver context to the consumers. From the consumer perspective this task can be called context acquisition. There are two methods to that are often used in context distribution:

\section{A. Balance of user control}

In order to increase software autonomy, applications depend on context information to dynamically adapt their Query: Context consumer makes a request in terms of a behavior to match the environment and user requirements query, so the context management system can use that [6]. Therefore, context-aware applications not only require query to produce results.

Subscription (also called publish / subscribe): Context middleware for distribution transparency of components, consumer can be allowed to subscribe with a context context-awareness. However, context-aware applications management system by describing the requirements. The may not always adapt as the user expects, and may cause system will then return the results periodically or when an users to feel loss of control over the behavior of their event occurs (threshold violation). In other terms, application.

consumers can subscribe for a specific sensor or to an event.

\section{B. Characteristics of Context Information}

Several requirements have to be taken into account when modeling context information [15]:

Heterogeneity and mobility:Context data obtained from databases or digital libraries like geographic map data are often static. Many context-aware applications are also mobile or depend on mobile context information sources. This adds to the problem of heterogeneity as the context information provisioning must be adaptable to the changing environment. Also, location and spatial layout of the context information play important roles due to this requirement.

Relationships and dependencies: There exist various relationships between types of context information that have to be captured to ensure correct behavior of the applications. One such relationship is dependency whereby context information entities/facts may depend on other context information entities.

Timeliness:Context-aware applications may need access to past states and future states (prognosis). Therefore, timeliness (context histories) is another feature of context information that needs to be captured by context models.

Imperfection:Due to its dynamic and heterogeneous nature, context information may be of variable quality. In fact, it may even be incorrect. Most sensors feature an inherent inaccuracy and the sensed values age if the physical world changes, so that this inaccuracy increases over time. Thus, a good context modeling approach must

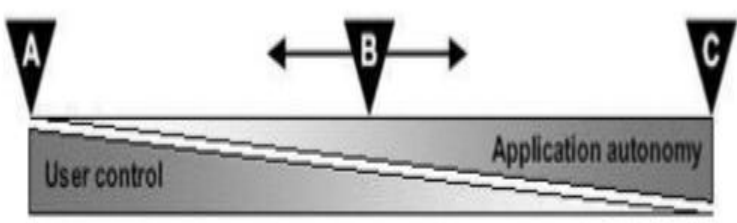

Fig 7. Continuum of user control versus software autonomy However we can see in figure, at the leftmost end (A), users is given full control over application behavior, and applications have very little autonomy. Applications designed in this way are the most interactive. Conversely, at the other end (C), applications only require a small amount of user control. Applications can also occupy any intermediate position on the continuum (position B). The appropriate position along the continuum will be dictated by the user's needs, situation and expertise.

But, context-aware applications may not always adapt as the user expects, and may cause users to feel loss of control over the behavior of their applications. Thus, an autonomous context-aware system must provide mechanisms to strike a suitable balance between user control and software autonomy.

In traditional applications, the trade-off between user control and software autonomy has been fixed at designtime. In contrast, context-aware applications may need to adjust the balance of user control and software autonomy at run-time by adjusting the level of feedback to users and the amount of user input. 


\section{B. Privacy}

Privacy is the right of the individual to control his personal information by posing specific obligations on the subjects that process his data. Privacy sensitive information is available to pervasive service providers continuously making it difficult to protect it. Stealing sensitive information through analysis of traffic might be constrained with use of cryptography but it is still possible to glean some information like identity, location and activities. Under any situation it is important to ensure that users don't feel they are being spied on and using the
services exposes them to unexpected threats and misuse of context and private information.

New technologies like RFID (Radio Frequency Identification) have on one hand made pervasive computing a reality but on the other hand have caused concerns about privacy as users are tracked when they move around. Laws such as the Patriot Act are also causing concerns that not only can hackers but also law enforcement agencies can intrude on the privacy of
individuals. Protection of private and sensitive information is mandated under laws such as HIPAA (Health Insurance techniques for deploying, configuring and managing Portability and Accountability Act) and the European networks of sensors [21][22].

Union's Data Protection Directive, but their Support for heterogeneity: hardware components ranging implementation in the Pervasive Computing realm is from resource-poor sensors, actuators and mobile client complicated ([16], [17]).

Privacy of location information deals with controlling must a variety of networking interfaces and programming access to the huge amount of sensitive information that language.

might be generated when location systems continuously Support for mobility: all components can be mobile and track users [18]. The user would not want to stop all access the communication protocols must therefore support because some applications can use this information to appropriately flexible forms of routing. Context provide critical services like public safety, transportation, information may need to migrate with context-aware emergency response, and disaster management [19], but components.

there is a need for the user to be in control [18].

\section{Security}

Scalability: context processing components and communication protocol must perform adequately in very changing domains.

When it comes to security, one size does not fit all [20]. Support for privacy: flows of context information between Hence, the security architecture deployed should be able to the distributed components of a context-aware system provide different levels of security services based on must be controlling according to user's privacy needs and system policy, context information, environmental expectations.

situations, temporal circumstances, available resources, Tolerance for component failures: sensors are likely to etc. Scenarios which require a higher-level of assurance or fail in the ordinary operation of a context-aware system; greater security may require users to interact with the disconnection may also occur.

security subsystem explicitly by, say, authenticating Ease of deployment and configuration: it must be easily themselves using a variety of means to boost system's deployed and configured to meet user and environmental confidence.

requirements.

Often, traditional security is somewhat static and context Dynamic reconfiguration: detecting changes in available insensitive. Pervasive computing integrates context and resources and reallocating them or notify the application to situational information, transforming the computing change its behavior.

environment into a sentient space. The security aspects of Adaptivity: the ability of a system to recognize unmet it are no exceptions. Security services should make needs within its execution context and to adapt itself to extensive use of context information available. For meet those needs.

example, access control decisions may depend on time or Asynchronous paradigm: decoupling the client and server special circumstances. Context data can provide valuable components and delivering multicast messages.

information for intrusion detection mechanisms. The principal of "need to know" should be applied on temporal and situational basis. For instance, security policies should be able to change dynamically to limit the permissions to the times or situations when they are needed. However, viewing what the security policy might become in a

\section{VI.CONCLUSION}

The current state-of-the-art of context-aware middleware explores quite different approaches to support pervasive and mobile computing based on context information. With the rapid development of information 
technology, it is inevitable that the distributed mobile computing will evolve to pervasive computing. The shift to the pervasive computing paradigm brings forth new challenges of security and privacy.

Based on our survey we conclude that context-aware middleware is the only middleware that can meet the new requirements imposed by mobility as it can sense the user activity continuously. In our future work we propose to provide dynamic reconfiguration to the context-aware middleware so that it can adapt and reconfigure itself to enhance the behavior of the application. This will help designers building mobile applications to better utilize the scarce resources of mobile device like CPU power, battery life and memory.

\section{REFERENCES}

[1] Linthicum D., B2B Application Integration: e-Business-Enable Your Enterprise, 2001

[2] Umar A., e-Business and Distributed Systems Handbook: Middleware Module. 2nd ed. NGE Solutions, 2004.

[3] Salber D., Dey A., Abowd G., "The Context Toolkit: Aiding the Development of Context-Enabled Applications", in Proceedings of ACM SIGHI Conference on Human Factors in Computing Systems (CHI-99), Pittsburgh, Pennsylvania, USA, 1999.

[4] Carpa L. et al.: Middleware for Mobile Computing, InProceedings of the 8th Workshop on Hot Topics in Operating Systems, Elmau, Germany, 2001

[5] Abowd G. D., Dey A. K., Brown P. J., Davies N., Smith M., and Steggles P., "Towards a better understanding of context and context-awareness," in Proceedings of the 1st international symposium on Handheld and Ubiquitous Computing, ser. HUC '99.London, UK: Springer-Verlag, 1999, pp. 304-307.

[6] Marco B. and Leonardo B., "A survey about context-aware middleware" $\quad$ Retrieved from http://www.slideshare.net/LeoBruni/a-survey-about-contextawaremiddleware, June 16, 2009.

[7] Henricksen K., "A framework for context-aware pervasive computing applications," Computer Science, School of Information Technology and Electrical Engineering, The University of Queensland, September 2003, http://henricksen.id.au/publications/phd-thesis.pdf [Accessed on:2012-01-05].

[8] Perera C., Zaslavsky A., Christen P. and Georgakopoulos D., "Context Aware Computing for The Internet of Things: A Survey", published in Communications Surveys \& Tutorials, IEEE (Volume:16, Issue: 1), 03 May 2013.

[9] Huebscher M. C., McCann J. A., "Adaptive middleware for contextaware applications in smarthomes", Published in Proceedings MPAC '04 Proceedings of the 2nd workshop on Middleware for pervasive and ad-hoc computing, Pages 111-116.

[10] Bernardos A., Tarrio P., and Casar J., "A data fusion framework for context-aware mobile services," in Multisensor Fusion and Integration for Intelligent Systems, 2008. MFI 2008. IEEE International Conference on, aug. 2008, pp. $606-613$

[11] Bikakis A., Patkos T., Antoniou G. and Plexousaki D., "A survey of semantics-based approaches for context reasoning in ambient intelligence," in Ambient Intelligence 2007 Workshops, M. M, F. A, and A. E, Eds., vol. 11. Springer-verlag berlin, 2008.

[12] Guan D., Yuan W., Lee S., and Lee Y.K., "Context selection and reasoning in ubiquitous computing," in Intelligent Pervasive Computing, 2007. IPC. The 2007 International Conference on, oct. 2007, pp. $184-187$. [Online].

[13] Nurmi P. and Floréen P., "Reasoning in Context-Aware Systems." Position paper Department of Computer Science, University of Helsinki., Dec 2004

[14] Hall D. and Llinas J., "An introduction to multisensor data fusion," Proceedings of the IEEE, vol. 85, no. 1, pp. 6-23,jan 1997.

[15] Shehzad A., Ngo H.Q., Pham K.A., Lee S. Y., "Formal Modeling in Context Aware Systems", In Proceedings of the $1 \mathrm{st}$ International Workshop on Modeling and Retrieval of Context (MRC'2004).
[16] Stanford V., "Pervasive health care applications face tough security challenges", Pervasive Computing, IEEE, Volume 1, Issue 2, April-June 2002, pp. 8-12.

[17] Jacobs A. R., Abowd G. D., "A Framework for comparing perspectives on privacy and pervasive technologies", Pervasive Computing, IEEE, Volume 2, Issue 4, Oct-Dec 2003, pp. 78-84

[18] Beresford A. R., Stajano F., "Location Privacy in Pervasive Computing", Pervasive Computing, IEEE, Volume 2, Issue 1, JanMar2003,pp.46-55.

[19] Lee C.K., Lee W.C., Leung H.V., "Nearest Surrounder Search", IEEE International Conference on Data Engineering, April 2006.

[20] Campbell R., Al-Muhtadi J., Naldurg P., Sampemane G., and Dennis Mickunas M.,"Towards Security and Privacy for Pervasive Computing" ISSS'02 Proceedings of the 2002 Mext-NSF-JSPS international conference on Software security: theories and systems, pages $1-15$

[21] Hadim S., Al-Jaroodi J. and Mohamed N., "Trends in middleware for mobile ad hoc networks", JCM,1(4):11-21, 2006

[22] Henricksen K., Indulska J., McFadden T. and Balasubramaniam S., "Middleware for distributed context-aware systems", In International Symposiumon Distributed Objects and Applications (DOA, pages 846-863. Springer, 2005

\section{BIOGRAPHIES}

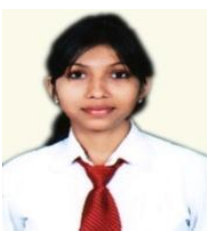

Miss. Chitra Sahuis PG Scholar. She holds B.E in Computer Engineering and pursuing her M.Tech from ShriRamdeobaba College of Engineering and Management, Nagpur. Her areas of interests are Computer Networks, Context Aware Middleware, Pervasiveand Ubiquitous Computing.

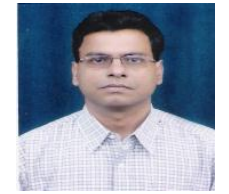

Dr.D. S. Adane is currently working as Professor and Head, Information Technology Department, Shri Ramdeobaba College of Engineering and Management, Nagpur. $\mathrm{He}$ is $\mathrm{PhD}$ in Computer Science and Engineering and has over 22 years of experience in teaching and research. His areas of interest are Distributed and Mobile Computing, Wireless Sensor Networks, Mobile Agents and Network Security. $\mathrm{He}$ is also Life member of ISTE and Institution of Engineers India (MIE). 